\title{
Successful management of seven cases of critical COVID-19 with early noninvasive-invasive sequential ventilation algorithm and bundle pharmacotherapy
}

Mian Peng ${ }^{1}$, Xueyan Liu $(\bowtie)^{2}$, Jinxiu $\mathrm{Li}^{3}$, Di Ren ${ }^{4}$, Yongfeng Liu ${ }^{5}$, Xi Meng ${ }^{3}$, Yansi Lyu ${ }^{6}$, Ronglin Chen ${ }^{5}$, Baojun $\mathrm{Yu}^{7}$, Weixiong Zhong ${ }^{1}$

${ }^{1}$ Department of Intensive Care Unit, The Third Affiliated Hospital of Shenzhen University, Shenzhen 518001, China; ${ }^{2}$ Department of Intensive Care Unit, Shenzhen People's Hospital, First Affiliated Hospital of Southern University of Science and Technology, The Second Affiliated Hospital of Jinan University, Shenzhen 518020, China; ${ }^{3}$ Department of Intensive Care Unit, The Third People's Hospital of Shenzhen, Shenzhen 518112, China; ${ }^{4}$ Department of Intensive Care Unit, The Second People's Hospital of Shenzhen, Shenzhen 518035, China; ${ }^{5}$ Department of Intensive Care Unit, Shenzhen Longgang Central Hospital, Shenzhen 518116, China; ${ }^{6}$ Department of Dermatology, Shenzhen University General Hospital, Shenzhen 518055, China; ${ }^{7}$ Department of Intensive Care Unit, Shenzhen Baoan District People's Hospital, Shenzhen 518101, China

(C) Higher Education Press 2020

\begin{abstract}
We report the clinical and laboratory findings and successful management of seven patients with critical coronavirus disease 2019 (COVID-19) requiring mechanical ventilation (MV). The patients were diagnosed based on epidemiological history, clinical manifestations, and nucleic acid testing. Upon diagnosis with COVID-19 of critical severity, the patients were admitted to the intensive care unit, where they received early noninvasive-invasive sequential ventilation, early prone positioning, and bundle pharmacotherapy regimen, which consists of antiviral, anti-inflammation, immune-enhancing, and complication-prophylaxis medicines. The patients presented fever $(n=7,100 \%)$, dry cough $(n=3,42.9 \%)$, weakness $(n=2,28.6 \%)$, chest tightness $(n=1$, $14.3 \%)$, and/or muscle pain $(n=1,14.3 \%)$. All patients had normal or lower than normal white blood cell count/ lymphocyte count, and chest computed tomography scans showed bilateral patchy shadows or ground glass opacity in the lungs. Nucleic acid testing confirmed COVID-19 in all seven patients. The median MV duration and intensive care unit stay were 9.9 days (interquartile range, 6.5-14.6 days; range, 5-17 days) and 12.9 days (interquartile range, 9.7-17.6 days; range, 7-19 days), respectively. All seven patients were extubated, weaned off MV, transferred to the common ward, and discharged as of the writing of this report. Thus, we concluded that good outcomes for patients with critical COVID-19 can be achieved with early noninvasive-invasive sequential ventilation and bundle pharmacotherapy.
\end{abstract}

Keywords COVID-19; noninvasive-invasive sequential ventilation; bundle pharmacotherapy

\section{Introduction}

The transmission of pathogens from animals to humans and resultant zoonotic infectious diseases have been associated with several major global outbreaks and constitute global public health burden. Generally, coronaviruses (CoVs) are enveloped RNA viruses that can cause respiratory and intestinal infections in humans and other animals. Human infections with $\mathrm{CoVs}$ had been associated with mild upper respiratory disease before the 2003

Received March 13, 2020; accepted April 17, 2020

Correspondence: Xueyan Liu, liuxueyan100916@163.com outbreak of severe acute respiratory syndrome (SARS) [1]. However, the discovery that SARS is caused by a new $\mathrm{CoV}$, SARS-CoV, redefined our understanding of CoVs [2]. The potential pathogenicity of CoVs was further elucidated by the 2012 Middle East respiratory syndrome (MERS) outbreak, which is attributed to infection with yet another new $\mathrm{CoV}$, MERS-CoV. MERS, which presents as acute pneumonia and occasional renal failure in severe cases, had an alarming 30\% mortality rate in the 2012 outbreak [3]. The severe morbidities of $\mathrm{CoV}$ diseases over the past two decades have shown that CoVs represent a serious infectious disease concern.

In December of 2019, a cluster of patients with pneumonia of unknown cause was linked to transmission 
at a seafood wholesale market in Wuhan, China. A previously unknown $\beta-\mathrm{CoV}$ was discovered in samples from affected patients. The 2019 novel CoV causing the ongoing pneumonia pandemic was identified to be of the same genus as the SARS-CoV and the seventh CoV known to infect humans following 229E, NL63, OC43, HKU1, MERS-CoV, and SARS-CoV [4,5]. This causative virus was named as severe acute respiratory syndrome coronavirus 2 (SARS-CoV-2). The relevant infectious disease was named as coronavirus disease 2019 (COVID-19) by the World Health Organization [6].

The cases of COVID-19 can be classified as mild, moderate, severe, or critical based on clinical symptoms and radiologic images [7]. A total of 87137 confirmed cases globally, including 79968 confirmed cases in China and 7169 outside China, with 2977 deaths (3.4\%) had been reported by the World Health Organization as of March 1, 2020 [8]. SARS-CoV-2 exhibits rapid transmission and causes atypical clinical symptoms, including fever, cough, myalgia, weakness, and dyspnea, as well as clinical signs recognizable in chest images that may be helpful for the early detection of emergent COVID-19 [9].

Patients with COVID-19 who become critically ill and require mechanical ventilation (MV) have a particularly high mortality risk. Therefore, an effective standard treatment regimen for COVID-19 that can limit disease severity should be established to quell public panic. Here, we report the successful management of seven critically ill patients diagnosed with COVID-19 who suffered acute respiratory failure.

\section{Case report}

We collected the clinical and laboratory data of the seven critically ill patients admitted to the intensive care unit (ICU) of the Third People's Hospital of Shenzhen.
Respiratory specimens (nasopharyngeal swabs, sputum, or bronchoalveolar lavage) from each patient were tested for SARS-CoV-2 with real-time reverse-transcriptase polymerase chain reaction testing.

The patients who presented the clinical symptoms of COVID-19 sought care in our hospital from January 20 to February 6, 2020. Suspected diagnoses of COVID-19 were made based on clinical symptoms and then confirmed based on white blood cell count (WCC)/lymphocyte count, chest computed tomography (CT) images, and nucleic acid test results. The patients were treated in the ICU for COVID-19. At present, these seven critically ill patients who suffered respiratory failure and required MV were treated successfully and discharged.

The seven critically ill patients (four women; 57.1\%), who are the subjects of this case series, had a median age of 56 years (interquartile range (IQR), 41-65 years; range, 36-71 years). The group included four patients (57.1\%) with body mass index $>25$, three patients with preexisting chronic diseases $(42.9 \%)$, and six patients with Wuhan contact history (85.7\%). All seven patients had fever $(100 \%)$, three had dry cough $(42.9 \%)$, two experienced weakness $(28.6 \%)$, one had chest tightness $(14.3 \%)$, and one reported muscle pain $(14.3 \%)$. The demographic and clinical characteristics of these patients are summarized in Table 1. Laboratory tests (results reported in Table 2) showed that all seven patients (100\%) had normal or lower than normal WCCs and lymphocyte counts, as well as bilateral patchy shadows or ground glass opacity in the lungs visible in chest CT scans (Fig. 1).

All the patients $(100 \%)$ were treated with the following bundle pharmacotherapy regimen: lopinavir/ritonavir tablets $(500 \mathrm{mg}$ every $12 \mathrm{~h}$ ) plus $\alpha$-interferon (5.0 MU atomized inhalation every $12 \mathrm{~h}$ ) as an antiviral treatment, thymosin $\alpha 1 \quad(1.6 \mathrm{mg} /$ day subcutaneous injection) to enhance immunity, methylprednisolone $(40 \mathrm{mg}$ /day for the first 3 days) to reduce pulmonary exudation, traditional

Table 1 Patients' demographic and clinical characteristics

\begin{tabular}{|c|c|c|c|c|c|c|c|}
\hline \multirow{2}{*}{ Characteristic } & \multicolumn{7}{|c|}{ Patient } \\
\hline & 1 & 2 & 3 & 4 & 5 & 6 & 7 \\
\hline Age (year) & 65 & 57 & 64 & 46 & 56 & 71 & 36 \\
\hline Gender & $\mathrm{F}$ & $\mathrm{F}$ & $\mathrm{F}$ & M & M & M & $\mathrm{F}$ \\
\hline Body mass index & 22 & 23 & 21 & 25 & 25 & 25 & 28 \\
\hline Underlying disease & Diab, 15 years & No & No & No & Hypt, 30 years & Hypt, 6 months & No \\
\hline Wuhan contact & Yes & Yes & Yes & Yes & No, but subway worker & Yes & Yes \\
\hline \multicolumn{8}{|l|}{ Symptoms } \\
\hline Fever & + & + & + & + & + & + & + \\
\hline Dry cough & + & - & - & + & - & - & + \\
\hline Weakness & - & - & - & + & + & - & - \\
\hline Chest tightness & + & - & - & - & - & - & - \\
\hline Muscle pain & - & - & - & + & - & - & - \\
\hline
\end{tabular}

F, female; M, male; Diab, diabetes; Hypt, hypertension. 
Table 2 Patients' laboratory and CT manifestation findings

\begin{tabular}{|c|c|c|c|c|c|c|c|}
\hline \multirow{2}{*}{ Parameter } & \multicolumn{7}{|c|}{ Patient } \\
\hline & 1 & 2 & 3 & 4 & 5 & 6 & 7 \\
\hline$\overline{\mathrm{WCC}}\left(\times 10^{9} / \mathrm{L}\right)$ & 5.89 & 9.14 & 5.39 & 6.64 & 6.51 & 5.64 & 3.81 \\
\hline Neutrophil count $\left(\times 10^{9} / \mathrm{L}\right)$ & 5.35 & 7.34 & 4.25 & 4.38 & 5.36 & 3.98 & 2.37 \\
\hline Lymphocyte count $\left(\times 10^{9} / \mathrm{L}\right)$ & 0.42 & 1.42 & 0.90 & 0.83 & 0.93 & 0.79 & 1.08 \\
\hline Hemoglobin $(g / L)$ & 118 & 143 & 125 & 159 & 124 & 142 & 92 \\
\hline Platelet $\left(\times 10^{9} / \mathrm{L}\right)$ & 118 & 175 & 176 & 171 & 153 & 191 & 282 \\
\hline $\mathrm{PaO}_{2} / \mathrm{FiO}_{2}$ ratio before intubation $(\mathrm{mmHg})$ & 136 & 165 & 118 & 150 & 116 & 110 & 141 \\
\hline Lactate $(\mathrm{mmol} / \mathrm{L})$ & 2.7 & 2.3 & 2.1 & 2.6 & 2.6 & 1.9 & 1.4 \\
\hline Creatinine $(\mu \mathrm{mol} / \mathrm{L})$ & 54.1 & 38.3 & 54 & 86 & 94.6 & 78.9 & 42 \\
\hline Urea $(\mathrm{mmol} / \mathrm{L})$ & 4.48 & 3.71 & 6.35 & 4.36 & 8.48 & 5.78 & 4.13 \\
\hline Albumin $(\mathrm{g} / \mathrm{L})$ & 34.6 & 37.9 & 39 & 46.6 & 35.5 & 40.8 & 41.3 \\
\hline Alanine aminotransferase (U/L) & 15.7 & 19.7 & 33.3 & 12 & 34.8 & 26.8 & 15 \\
\hline Total bilirubin $(\mu \mathrm{mol} / \mathrm{L})$ & 8.4 & 12.1 & 10.8 & 11.9 & 12.2 & 33.6 & 7.7 \\
\hline CT lung changes & + & + & + & + & + & + & + \\
\hline Respiratory nucleic acid result & + & + & + & + & + & + & + \\
\hline
\end{tabular}
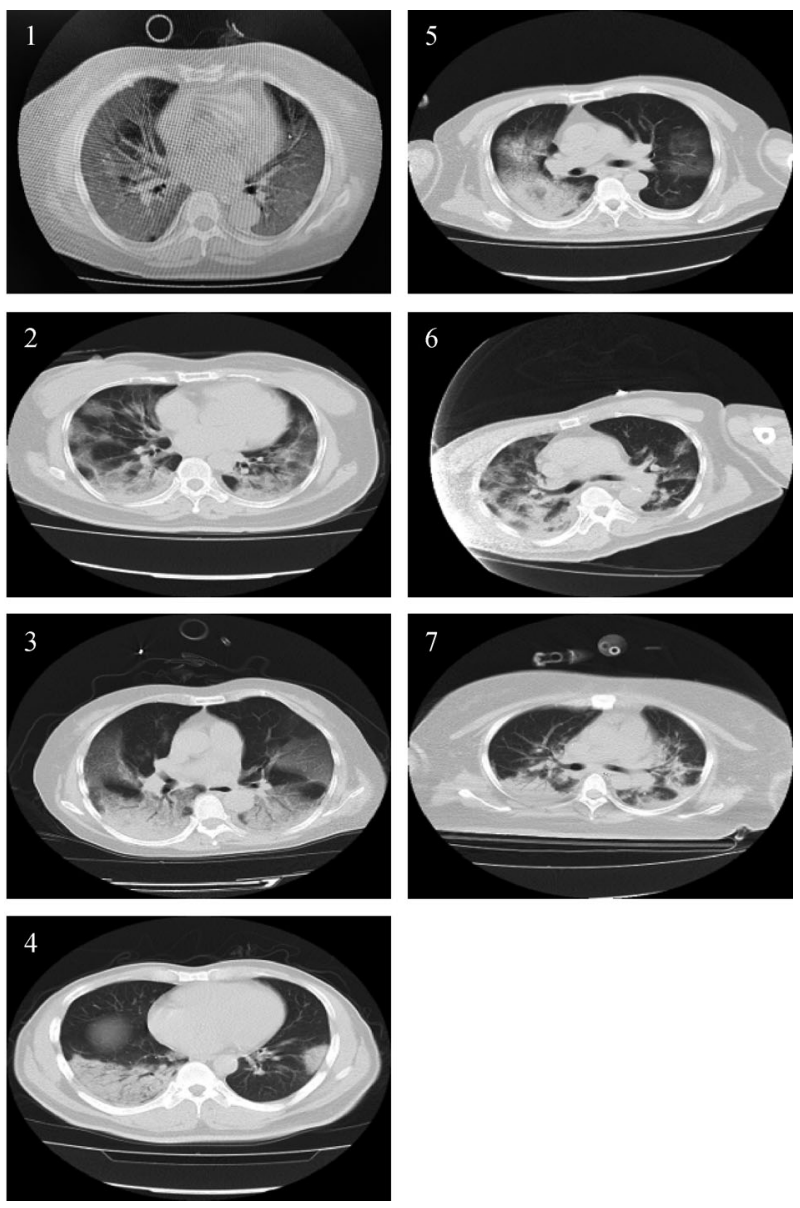

Fig. 1 Pretreatment chest CT scans for patients 1-7. Patient numbers are indicated in the upper left of each image.
Chinese medicine Xuebijing (100-mL injection every $12 \mathrm{~h}$ ) to attenuate inflammation, and low-molecular-weight heparin (4.0 kIU subcutaneous injection each day) to prevent deep vein thrombosis. The drugs (lopinavir/ ritonavir and $\alpha$-interferon) were stopped when two consecutive negative respiratory sample nucleic acid tests with a sampling interval of $\geqslant 1$ day were achieved.

The patients received noninvasive ventilation (NIV) with $60 \%$ oxygen absorption concentration $\left(\mathrm{FiO}_{2}\right)$ and inspired/expiratory positive airway pressure (IPAP/EPAP) levels of $8-12$ and $6 \mathrm{cmH}_{2} \mathrm{O}$, respectively. However, the patients' conditions were not relieved in $2 \mathrm{~h}\left(\mathrm{PaO}_{2} / \mathrm{FiO}_{2}\right.$ ratio reported in Table 2). Subsequently, all the patients were advanced to invasive MV with prone positioning (12-14 h/day) in the first 3-5 days. The median MV duration was 9.9 days (IQR, 6.5-14.6 days; range, 5-17 days). The MV start and end dates and duration for each patient, as well as ICU admission and transfer-out dates and duration of ICU stay, are reported in Table 3. None of the patients received extracorporeal membrane oxygenation.

All seven patients were extubated and weaned off their ventilators by February 25, 2020 and exhibited clinically smooth breathing without fever or any other COVID-19 symptoms. Additionally, chest CT scans showed absorption of the aforementioned lung changes in all cases (Fig. 2). The patients were thus transferred from the ICU to the common ward. The patients had a median ICU stay of 12.9 days (IQR, 9.7-17.6 days; range, 7-19 days; Table 3) and were discharged as of the writing of this report. 


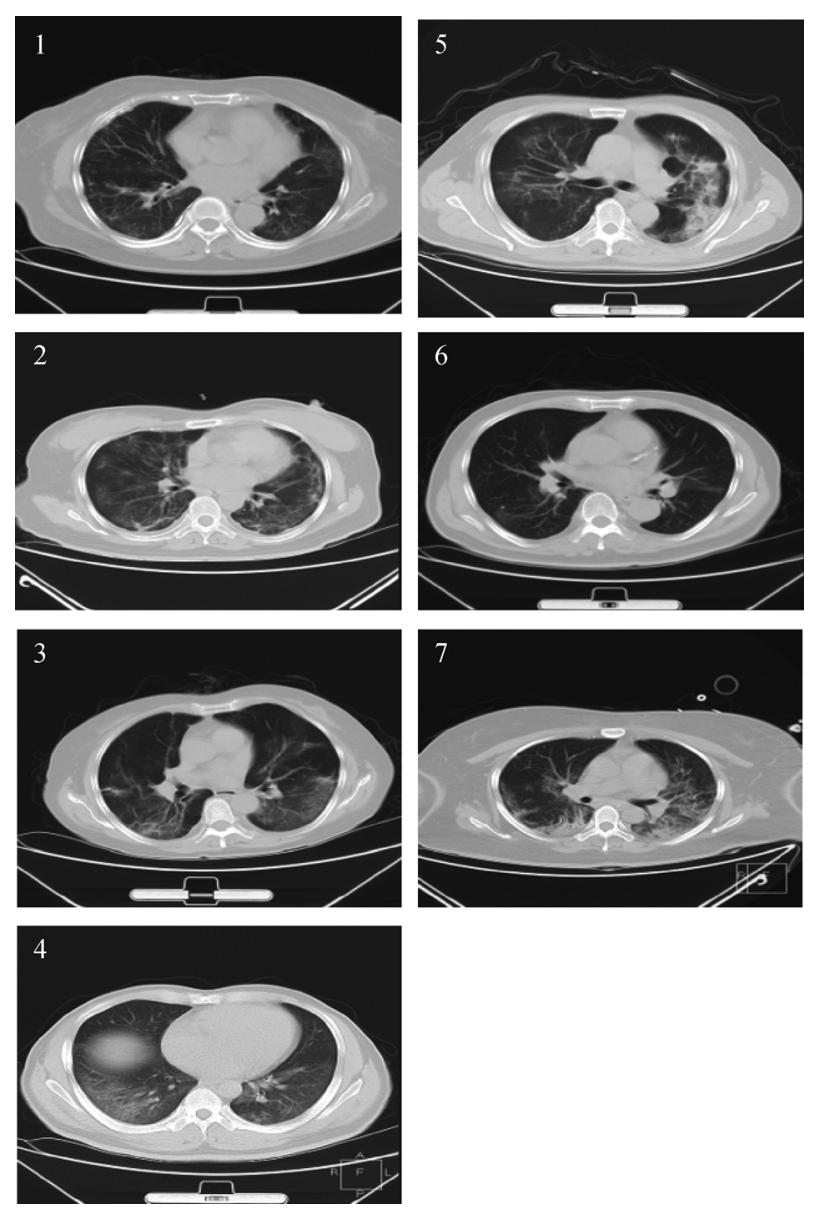

Fig. 2 Post-treatment chest CT scans for patients 1-7. Patient numbers are indicated in the upper left of each image.

\section{Discussion}

SARS-CoV-2, the underlying causative agent of COVID-19, can be transmitted from person to person; thus, SARS-CoV-2 and COVID-19 has rapidly spread $[10,11]$. Patients infected with SARS-CoV-2 have since been found in China, other countries in Asia, the Middle East, Europe, and North America [12]. Thus, an urgent international need for the establishment of effective therapeutic strategies has emerged to achieve the rapid control of SARS-CoV-2 infection and COVID-19.

According to China's national guidelines for the diagnosis of COVID-19 (summarized in Fig. 3) [7], these seven cases were diagnosed with COVID-19 because of the positive nucleic acid affirmation of SARS-CoV-2 in their respiratory samples (Table 2). COVID-19 can be classified as mild, moderate, severe, or critical. Mild COVID-19 is characterized by mild clinical symptoms without signs of pneumonia on imaging. Moderate COVID-19 is characterized by fever, respiratory symptoms, and imaging manifestations of pneumonia. Moderate-type COVID-19 is elevated to severe-type if the patient shows respiratory distress (defined by a respiratory rate of $\geqslant 30 \mathrm{breaths} / \mathrm{min}$, a resting oxygen saturation rate of $\leqslant 93 \%$, or a partial arterial oxygen pressure $\left(\mathrm{PaO}_{2}\right) /$ $\mathrm{FiO}_{2} \leqslant 300 \mathrm{mmHg}$ ). Severe COVID-19 is considered to have progressed to critical COVID-19 if the patient develops respiratory failure requiring $\mathrm{MV}$, sepsis, or combined with organ failure requiring ICU monitoring and care. The seven cases of COVID-19 presented in this paper exhibited respiratory failure requiring invasive MV and thus were classified as critical-type COVID-19.

Our department is focused on the early diagnosis and severity typing of COVID-19. Our management plan upon confirmation of a COVID-19 diagnosis includes the immediate treatment of symptoms and the delivery of a comprehensive bundle pharmacotherapy. Monitoring and supportive treatment is usually sufficient in patients with mild or moderate COVID-19 to ensure good outcome. We provide close monitoring and bundle pharmacotherapy, as well as supplemental oxygen therapy, in patients with

Table 3 Patients' MV care spans, ICU admission spans, and notable case characteristics

\begin{tabular}{|c|c|c|c|c|c|c|c|}
\hline \multirow{2}{*}{ Parameter } & \multicolumn{7}{|c|}{ Patient } \\
\hline & 1 & 2 & 3 & 4 & 5 & 6 & 7 \\
\hline MV start date (in 2020) & Jan 24 & Jan 27 & $\operatorname{Jan} 31$ & Feb 1 & Feb 3 & Feb 4 & Feb 6 \\
\hline MV end date (in 2020) & Feb 1 & Feb 12 & Feb 7 & Feb 6 & Feb 10 & Feb 13 & Feb 23 \\
\hline MV duration (day) & 8 & 16 & 7 & 5 & 7 & 9 & 17 \\
\hline ICU admission date (in 2020) & Jan 24 & Jan 26 & Jan 28 & Feb 1 & Feb 3 & Feb 4 & Feb 6 \\
\hline ICU transfer out date (in 2020) & Feb 6 & Feb 13 & Feb 9 & Feb 8 & Feb 12 & Feb 16 & Feb 25 \\
\hline ICU stay (day) & 13 & 18 & 12 & 7 & 9 & 12 & 19 \\
\hline Notable case characteristics & $\begin{array}{l}\text { Sulp for } \\
\uparrow \text { sputum, } \\
\text { cultures } \\
\text { negative }\end{array}$ & $\begin{array}{l}\text { VAP; sepsis; } \\
\text { NE; sputum; } \\
\text { culture } P m, \\
\text { ESBL + E. } \\
\text { coli; AB: Taz, } \\
\text { Mer, Cef }\end{array}$ & - & $\begin{array}{l}\text { Sulp for } \\
\uparrow \text { sputum, } \\
\text { cultures } \\
\text { negative }\end{array}$ & $\begin{array}{l}\text { Sulp for } \\
\uparrow \text { sputum, } \\
\text { cultures } \\
\text { negative }\end{array}$ & - & $\begin{array}{l}\text { VAP; sputum } \\
\text { culture MDR } \\
\text { Pm; AB: Mer, } \\
\text { Taz, Ami }\end{array}$ \\
\hline
\end{tabular}

Jan, January; Feb, February; Sulp, sulperazone; VAP, ventilator-acquired pneumonia; NE, norepinephrine (low-dose acute treatment in this case); Pm, Pseudomonas; ESBL +, extended spectrum beta-lactamase positive; AB, antibiotic drugs administered; Taz, tazocin; Mer, meropenem; Cef, ceftazidime; MDR, multi-drug resistant; Ami, amikacin. 


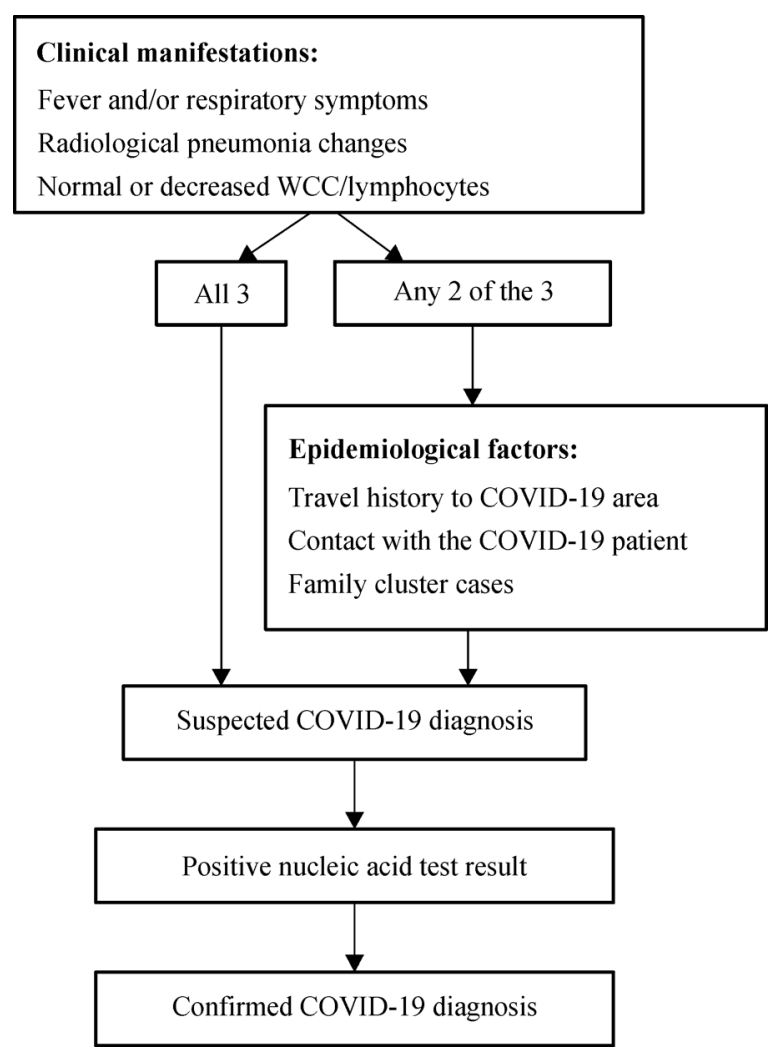

Fig. 3 COVID-19 diagnoses algorithm.

severe COVID-19 to avoid the progression of COVID-19 to critical level, which can be fatal. Therapy plans may be individualized depending on the particular characteristics of individual patients (Table 3 ).

The patients with critical COVID-19 were weaned off MV successfully, transferred to the common ward, and subsequently discharged. Our experience with the concentrated treatment of this patient population from Shenzhen, China has yielded three noteworthy lessons explained below.

First, we found that the early determination of COVID19 severity is of utmost importance. Missed diagnosis and misdiagnosis caused by atypical symptomology results in the loss of opportunity for early intervention [9]. Patients with the following factors are at risk of severe or critical COVID-19 [13,14]: age > 60 years; body mass index > 25; underlying chronic disease; low lymphocyte count $\left(<0.95 \times 10^{9} / \mathrm{L}\right)$; and nasopharyngeal swab viral nucleic acid value $<30$ or positive results with blood, stool, urine, or tear specimen. We believe that the most important among these factors may be lymphopenia. Three among the seven patients described in this report were over 60 years old with a chronic underlying disease and lymphocyte counts $<0.95 \times 10^{9} / \mathrm{L}$, which indicate a less than robust immunocompetence. Patients with lymphopenia tend to have the most serious symptoms, especially severe hypoxemia, and are prone to developing sepsis or multiple organ failure.

Second, early sequential noninvasive-invasive respiratory support and early prone position are effective measures for improving outcomes. Respiratory support is a very important technique that can save patients with severe COVID-19 who suffer from respiratory failure by improving oxygenation to reduce mortality. Therefore, appropriate respiratory support technique is the key point related to its success or failure [15]. Early respiratory support can facilitate disease recovery and improve prognosis [16]. We have come to favor a sequential noninvasive-invasive ventilation algorithm (summarized in Fig. 4), wherein patients with COVID-19 are started on NIV (IPAP, 8-12 $\mathrm{cmH}_{2} \mathrm{O}$; EPAP, $6 \mathrm{cmH}_{2} \mathrm{O}$; $\mathrm{FiO}_{2}, 60 \%$ ) if they have a $\mathrm{PaO}_{2} / \mathrm{FiO}_{2}$ ratio of $\leqslant 200 \mathrm{mmHg}$ (patients alert, hemodynamically stable). If the patients' symptoms showed no improvement after $2 \mathrm{~h}$ of NIV, then they are advanced to MV (parameters in Fig. 4). We restricted the duration of NIV to $\leqslant 2 \mathrm{~h}$ because respiratory failure cannot be relieved with NIV and the patients will continue to incur further oxygen debt if they have massive lung consolidation. MV with sedation and analgesia allows patients with critical COVID-19 to rest while giving time for our comprehensive pharmacotherapy regimen to exert therapeutic efficacy in the lungs. The patients were rested in prone position to ensure that the diaphragm is in a favorable position to enable its movement while reducing the pressure gradient in the chest cavity. This position

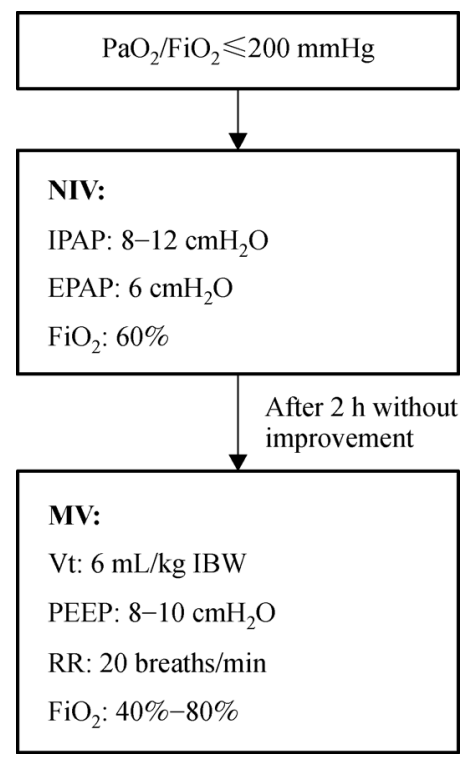

Fig. 4 Sequential noninvasive-invasive ventilation process. Vt, tidal volume; PEEP, positive end-expiratory pressure; RR, respiratory rate; IBW, ideal body weight. 
increases the functional residual capacity of the lungs, which improves oxygenation by improving the ventilationto-blood flow ratio and enhancing secretion drainage and fluid movement in the lungs.

Third, the administration of a bundle pharmacotherapy can support recovery from COVID-19. We recommend a bundle therapy (see case report text for dosage information) with three principal components: (1) antiviral (lopinavir/ ritonavir plus $\alpha$-interferon) and anti-inflammation drugs (methylprednisolone and Xuebijing), (2) immune system support (thymosin $\alpha 1$ ), and (3) prevention/treatment of complications (low-molecular-weight heparin). All seven patients were treated with this bundle pharmacotherapy while receiving respiratory support and recovered well. All of them have been discharged as of the writing of this report. Our discharge criteria for patients recovering from COVID-19 are: normal body temperature for $\geqslant 3$ days; alleviation of respiratory symptoms; majority resolution of radiological lung changes; and two consecutive negative respiratory sample nucleic acid tests with a sampling interval of $\geqslant 1$ day [7].

Among the seven critically ill patients whose cases are described in this report, five patients had MV durations of 5-9 days, and two patients required extended MV durations (16 and 17 days) because of ventilator-acquired pneumonia infections, which caused sepsis in one case (see Table 3 for bacteria identified from sputum cultures and individualized treatments). Consistent with the findings, we found that complications due to secondary infection are rare if the period of disease requiring $\mathrm{MV}$ is within a week or so. Conversely, patients requiring 2 weeks or more of MV become exposed to greater risk of acquiring negative bacilli, positive cocci, and fungal infections, which make the patients at increased risk of systemic infection, sepsis, multiple-organ dysfunction, and death. Thus, the strict control of hospital-acquired infections is of utmost importance in patients requiring $\mathrm{MV}$ to improve their likelihood of achieving rescue success. We aim to facilitate such success in part by enhancing patient immunity as discussed above.

This report has a notable limitation in that only seven cases of successfully treated critical ill patients with COVID-19 were included. More cases are needed to further confirm the effectiveness of our recommended treatment regimen. We hope that our treatment protocol will be applied to patients more broadly to collect data on its efficacy from broader populations.

\section{Conclusions}

Good treatment outcomes can be achieved for critically ill patients with COVID-19 with early sequential noninvasive-invasive ventilation and bundle pharmacotherapy, which comprises antiviral and anti-inflammation medicines, immune system support, and prophylaxis for vascular complications.

\section{Acknowledgements}

We acknowledge the writing guidance provided by Prof. Kunmei Ji and the training camp for medical research held by Shenzhen Medical Association and Huada. We are grateful to the physicians and nurses of the Third People's Hospital of Shenzhen who participated in the clinical examinations and sample collection.

\section{Compliance with ethics guidelines}

Mian Peng, Xueyan Liu, Jinxiu Li, Di Ren, Yongfeng Liu, Xi Meng, Yansi Lyu, Ronglin Chen, Baojun Yu, and Weixiong Zhong declare no conflict of interest. Written informed consent was obtained from all the patients.

\section{References}

1. Masters PS, Kuo L, Ye R, Hurst KR, Koetzner CA, Hsue B. Genetic and molecular biological analysis of protein-protein interactions in coronavirus assembly. Adv Exp Med Biol 2006; 581: 163-173

2. Drosten C, Günther S, Preiser W, van der Werf S, Brodt HR, Becker S, Rabenau H, Panning M, Kolesnikova L, Fouchier RA, Berger A, Burguière AM, Cinatl J, Eickmann M, Escriou N, Grywna K, Kramme S, Manuguerra JC, Müller S, Rickerts V, Stürmer M, Vieth S, Klenk HD, Osterhaus AD, Schmitz H, Doerr HW. Identification of a novel coronavirus in patients with severe acute respiratory syndrome. N Engl J Med 2003; 348(20): 1967-1976

3. Zaki AM, van Boheemen S, Bestebroer TM, Osterhaus AD, Fouchier RA. Isolation of a novel coronavirus from a man with pneumonia in Saudi Arabia. N Engl J Med 2012; 367(19): 18141820

4. Zhu N, Zhang D, Wang W, Li X, Yang B, Song J, Zhao X, Huang B, Shi W, Lu R, Niu P, Zhan F, Ma X, Wang D, Xu W, Wu G, Gao GF, Tan W; China Novel Coronavirus Investigating and Research Team. A novel coronavirus from patients with pneumonia in china, 2019. N Engl J Med 2020; 382(8): 727-733

5. Zhou P, Yang XL, Wang XG, Hu B, Zhang L, Zhang W, Si HR, Zhu Y, Li B, Huang CL, Chen HD, Chen J, Luo Y, Guo H, Jiang RD, Liu MQ, Chen Y, Shen XR, Wang X, Zheng XS, Zhao K, Chen QJ, Deng F, Liu LL, Yan B, Zhan FX, Wang YY, Xiao GF, Shi ZL. A pneumonia outbreak associated with a new coronavirus of probable bat origin. Nature 2020; 579(7798): 270-273

6. He F, Deng Y, Li W. Coronavirus disease 2019 (COVID-19): what we know? J Med Virol 2020; 92(7): 719-725

7. Lin L, Li TS. Interpretation of Guidelines for the Diagnosis and Treatment of Novel Coronavirus (2019-nCoV) Infection by the National Health Commission (Trial Version 5). Natl Med J China (Zhonghua Yi Xue Za Zhi) 2020; 100(11): 805-807 (in Chinese)

8. Guo YR, Cao QD, Hong ZS, Tan YY, Chen SD, Jin HJ, Tan KS, Wang DY, Yan Y. The origin, transmission and clinical therapies on coronavirus disease 2019 (COVID-19) outbreak - an update on the status. Mil Med Res 2020; 7(1): 11

9. Zhou L, Liu HG. Early detection and disease assessment of patients with novel coronavirus pneumonia. Chin J Tuberc Respir Dis 
(Zhonghua Jie He He Hu Xi Za Zhi) 2020; 43(3): 167-170 (in Chinese)

10. Jiang S, Du L, Shi Z. An emerging coronavirus causing pneumonia outbreak in Wuhan, China: calling for developing therapeutic and prophylactic strategies. Emerg Microbes Infect 2020; 9(1): 275277

11. Chan JF, Yuan S, Kok KH, To KK, Chu H, Yang J, Xing F, Liu J, Yip CC, Poon RW, Tsoi HW, Lo SK, Chan KH, Poon VK, Chan WM, Ip JD, Cai JP, Cheng VC, Chen H, Hui CK, Yuen KY. A familial cluster of pneumonia associated with the 2019 novel coronavirus indicating person-to-person transmission: a study of a family cluster. Lancet 2020; 395(10223): 514-523

12. Bogoch II, Watts A, Thomas-Bachli A, Huber C, Kraemer MUG, Khan K. Potential for global spread of a novel coronavirus from China. J Travel Med 2020; 27(2): taaa011

13. Expert Panel of Critical Care Medicine for COVID-19 Pneumonia in
Shenzhen. The Shenzhen 2020 guidelines for the diagnosis and treatment of severe/critical COVID-19. Chin Crit Care Med (Zhonghua Wei Zhong Bing Ji Jiu Yi Xue) 2020; 32(2): 1-4 (in Chinese)

14. Shi Y, Yu X, Zhao H, Wang H, Zhao R, Sheng J. Host susceptibility to severe COVID-19 and establishment of a host risk score: findings of 487 cases outside Wuhan. Crit Care 2020; 24(1): 108

15. Yuan X, Mu JS, Mo GX, Hu XS, Yan P, Xie LX. Respiratory support for severe 2019-nCoV pneumonia suffering from acute respiratory failure: time and strategy. Chin J Tuberc Respir Dis (Zhonghua Jie He He Hu Xi Za Zhi) 2020; 43(3): 177-180 (in Chinese)

16. Liu K, Fang YY, Deng Y, Liu W, Wang MF, Ma JP, Xiao W, Wang YN, Zhong MH, Li CH, Li GC, Liu HG. Clinical characteristics of novel coronavirus cases in tertiary hospitals in Hubei Province. Chin Med J (Engl) 2020; 133(9): 1025-1031 